\title{
Negative External Cost Computing Model for Noise of China's High-speed Railway and its Verification and Trend Analysis
}

\author{
Xiaomei Xuan ${ }^{1}{ }^{*}$ \\ ${ }^{1}$ Graduate Department, China Academy of Railway Science, Beijing 100081, China
}

\begin{abstract}
The purpose of this paper is to calculate the external noise cost of China's high-speed railway and forecast its future trend. Firstly, the unit value transfer method after correction is selected as a method of calculation. Secondly, the noise cost calculation model of China's high-speed railway is established by introducing the correction factors such as GDP-PPP, population density ratio and CPI ratio. At last, the external noise cost since the opening of high-speed railway is calculated. The model validation results show that although the average external noise cost of high-speed railway increases year by year, it does not increase significantly. This is not only related to economic growth and improvement of noise reduction technology, but also related to the increase of investment in noise reduction facilities in the early stage. Compared with it, the total external cost of high-speed railway noise increases obviously, which is positively related to the rapid development of high-speed railway. With the increase of high-speed railway mileage and passenger turnover, the total noise cost increases significantly. It can be predicted that with the implementation of high-speed railway planning in the future, the negative external cost of noise will continue to increase.
\end{abstract}

\section{Introduction}

As an important transportation infrastructure, high-speed railway provides convenient, efficient and safe services for the public, at the same time, it has produced huge social and economic benefits and international influence. In the stage of emphasizing economic development, positive externality is often easier to be concerned, but the increasingly prominent environmental problems will inevitably become the bottleneck of restricting economic development. When the ecological environment becomes a major social problem related to people's livelihood, negative externality will be highlighted. The general negative externalities of transportation system are mainly reflected in traffic congestion, traffic accidents, noise pollution, air pollution and climate change. Although compared with other means of transportation, high-speed railway has great advantages in environmental protection, it still inevitably brings negative impacts on the surrounding residents and local environment, including noise, electromagnetic radiation, environmental pollution during the construction period, traffic accidents, lack of fairness and unbalanced regional development. At present, the noise problem caused by high-speed railway has attracted great attention of the public, and has become a key factor restricting the speed-up and development of high-speed railway.

According to the externality theory, the reason for the negative externality of high-speed railway noise is that the noise of high-speed railway has an impact on others, and the latter pays extra cost but cannot be compensated. As a rational person, high-speed railway operators do not take noise and other external costs into account when making decisions, but only consider all the social costs caused by the provision and use of transport infrastructure. Because the negative external cost of high-speed railway noise is separated from the price system, it cannot be reflected in the market price, which leads to the imbalance of resource allocation efficiency and leads to the suboptimal result. High speed railway noise external cost accounting is to quantify and monetize the impact of noise on the environment, establish the calculation model of noise external cost, convert environmental indicators into economic indicators, and then realize the internalization of external environmental costs.

\section{Model building}

\subsection{Determine the calculation method}

\subsubsection{Background}

Combined with domestic and foreign research the main methods for quantifying the externality cost of transportation are damage cost approach, avoidance cost approach, replacement cost approach and unit/function value transfer approach.

After comparing the application scope of each method, and considering the actual situation of China, we choose the unit value transfer method as the calculation method of noise external cost. It's quite difficult to obtain effective crowd data of noise exposure on both sides of the railway

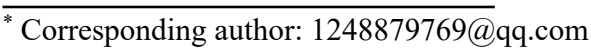


in a short time due to the dense high-speed railway network and long operation mileage in China. So, the unit transfer method should be the most convenient way to reflect the external noise cost of China's high-speed railway currently.

\subsection{2 the unit value transfer method}

Unit value transfer refers to the direct transfer of major data from the original location to a new location, which can be directly transferred or slightly adjusted. The European Union has studied the external cost of transportation earlier and issued three editions of the guidance manual on external cost [1]. Therefore, we choose the mature calculation results of EU as the unit value. For the economic differences and noise exposure population differences between EU28 countries and China, the corresponding correction factors, such as income, population density and other factors, can be selected to correct [2], and the model suitable for noise cost estimation of high-speed railway in China can be given as far as possible.

\subsection{Model parameter setting}

\subsubsection{Unit value}

The European Union has studied the external cost of transportation earlier and published a manual to guide the calculation of external cost of transportation. In view of its perfect data support, this paper selects the data of EU28 as a reference. According to the latest handbook (version 2019) published by EU, the average noise cost of highspeed railway in EU 28 countries in 2016 is selected as the unit value.

By comparing the national conditions of EU and China, the corresponding correction coefficient is selected to convert the average noise cost of EU28 high-speed railway in 2016 into the average noise cost of China's high-speed railway in the same year, and 2016 is taken as the base year to calculate the average noise cost of other years.

\subsubsection{Noise cost evaluation index}

The average noise cost and total noise cost of high-speed railway are selected as the evaluation indexes of noise externality cost of high-speed railway.

\subsubsection{Noise threshold}

The threshold value of railway noise is the same as that of other traffic modes. According to the recommended values in the book, the noise threshold is $50 \mathrm{~dB}(\mathrm{~A})$, and the damage caused by exceeding this value is included in the external cost of noise.

\subsubsection{Noise cost range}

The range of noise cost includes two aspects: one is annoyance value and the other is health value.

The annoyance value mainly comes from noise interference. Noise interference is the interference that people suffer from in traffic noise [3]. It will hinder people to carry out some activities and may lead to a variety of negative reactions, including anger, disappointment, anxiety, fatigue and sleep disorders. The annoyance value was calculated by WTP method. The health value comes from the diseases that can be caused by long-term and frequent exposure to noise, including ischemic heart disease, stroke, dementia, hypertension and so on [4][5]. The calculation of health value is based on the environmental burden of disease.

\subsubsection{Correction factor}

When using the unit value transfer method, we need to compare the information of the two countries and select the appropriate correction coefficient for correction. The noise annoyance cost involved in the model uses the method of willingness to pay for noise, and its value is related to the economic situation of countries and regions; The health cost of noise is not only related to economy, but also closely related to the number of exposed populations. To sum up, this paper selects the correction coefficient related to economy and population.

- Purchasing power parity (PPP) GDP ratio (GDP-PPP)

The difference of economic situation and income in different countries and regions will lead to different willingness to pay and different health costs. In order to eliminate the impact of exchange rate instability, GDPPPP ratio is chosen as the correction factor to minimize the cost difference.

-Population density ratio

The manual applies the data of exposed population on both sides of the railway trunk line in the EU noise map. Because there is no noise map management policy in China's railways, it is impossible to accurately obtain all the exposed population around the railway, so the population density ratio is introduced to evaluate the noise affected population.

\subsection{Noise cost calculation model}

\subsubsection{Calculation model of average noise cost}

The average noise cost of high-speed railway in each year is set as $C_{n}$. The average noise cost model of high-speed railway in China can be expressed as follows:

Where,

$$
C_{n}=e_{n} d_{p} g C_{E U 28-2016}
$$

$C_{n}$ - average noise cost of high-speed railway in each year $(\mathrm{n}=1-12)(2008-2019)$

$e_{n}$-the ratio of consumer price index in each year;

$d_{p}$-the ratio of population density

$g$-purchasing power parity GDP ratio

$C_{E U 28-2016-a v e r a g e}$ noise cost of EU28 high-speed railway in 2016 


\subsubsection{Calculation model of total noise cost}

The total noise cost of high-speed railway in each year is set as $C_{n}$ '. Then the total noise cost model of high-speed railway can be expressed as follows:

$$
C_{n}{ }^{\prime}=C_{n} P_{n}
$$

$C_{n}$ '-Total noise cost of high-speed railway in each year

$P_{n}$-High-speed railway passenger turnover

$\forall$ n- 1 12, Year2008 2019.

\subsection{Model variable calculation}

- $\mathrm{g}$

In 2016, China's GDP calculated by purchasing power parity (PPP) was 13572.62 (current international yuan), and that of EU28 in the same year was 40584.879 (current international yuan). It is calculated that $\mathrm{g}$ is equal to 0.3344 .

- $d_{p}$

The population density of China is 146.282 per square kilometre, and that of the European Union is 111.482 per square kilometre [6]. It is calculated that $d_{p}$ is equal to 1.3122 .

- $C_{E U 28-2016}$

In 2016, $C_{E U 28-2016}$ was equal to 0.3 Euro-cent $/ \mathrm{pkm}[1]$. the exchange rate of Euro to $\mathrm{RMB}$ was 7.1245 . So $C_{E U 28-}$ 2016 was equal to $2.1374 \mathrm{RMB}-\mathrm{cent} / \mathrm{pkm}$.

- $e_{n}$ and $P_{n}$

Annual value of $e_{n}$ and $P_{n}$ are shown in the table below.

Table 1. Annual value of $e_{n}$ and $P_{n}$.

\begin{tabular}{|c|c|c|c|}
\hline $\mathbf{n}$ & Year & $\boldsymbol{e}_{\boldsymbol{n}}$ & $\boldsymbol{P}_{\boldsymbol{n}}(\mathbf{1 0 0}$ million pkm) \\
\hline 1 & 2008 & 0.8330 & 15.6 \\
\hline 2 & 2009 & 0.8142 & 162.2 \\
\hline 3 & 2010 & 0.8543 & 463.2 \\
\hline 4 & 2011 & 0.8924 & 1058.4 \\
\hline 5 & 2012 & 0.9238 & 1446.1 \\
\hline 6 & 2013 & 0.9479 & 2141.1 \\
\hline 7 & 2014 & 0.9669 & 2825 \\
\hline 8 & 2015 & 0.9804 & 3863.4 \\
\hline
\end{tabular}

The basic data used in the above calculation are from the World Bank Database[6]and China Statistical Yearbook[7].

\section{Calculation results and trend analysis}

\subsection{Average cost of noise of China's high-speed railway}

This paper selects the relevant data of high-speed railway from 2008 to 2019 to verify the model. The average noise cost is obtained by substituting the data into the previous model. The results are shown in table 2 and Figure1.

Table2. Average noise cost of high-speed railway

\begin{tabular}{|c|c|}
\hline Year & $\begin{array}{c}\text { Average cost of noise } \\
\text { RMB-cent/pkm }\end{array}$ \\
\hline 2008 & 0.78 \\
\hline 2009 & 0.76 \\
\hline 2010 & 0.80 \\
\hline 2011 & 0.84 \\
\hline 2012 & 0.87 \\
\hline 2013 & 0.89 \\
\hline 2014 & 0.91 \\
\hline 2015 & 0.92 \\
\hline 2016 & 0.94 \\
\hline 2017 & 0.95 \\
\hline 2018 & 0.97 \\
\hline 2019 & 1.00 \\
\hline
\end{tabular}

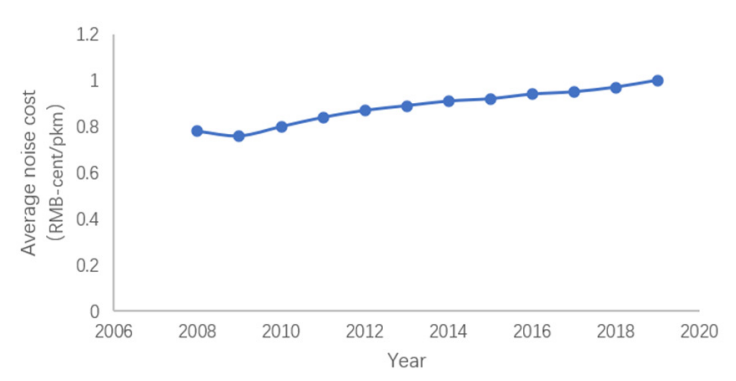

Fig. 1. Average noise cost of high-speed railway.

It can be seen from Figure 1 that during 2008-2019, the average noise cost of China's high-speed railway is increasing year by year, but the increase rate is slow. The increase of average noise cost is unlikely to be related to economic growth, technological progress, improvement of noise reduction technology level and increase of investment in noise reduction facilities in the early stage.

\subsection{The total cost of noise of China's high-speed railway}

According to the total noise cost model, the annual total noise cost of high-speed railway is calculated by using the average noise cost of high-speed railway and passenger turnover. The results are shown in Table 3 and Figure2.

Table3. The total noise cost of high-speed railway

\begin{tabular}{|c|c|}
\hline Year & $\begin{array}{c}\text { The total cost of noise } \\
\text { RMB100mn }\end{array}$ \\
\hline 2008 & 0.12 \\
\hline
\end{tabular}




\begin{tabular}{|l|l|}
\hline 2009 & 1.24 \\
\hline 2010 & 3.71 \\
\hline 2011 & 8.86 \\
\hline 2012 & 12.53 \\
\hline 2013 & 19.04 \\
\hline 2014 & 25.62 \\
\hline 2015 & 35.52 \\
\hline 2016 & 43.53 \\
\hline 2017 & 55.98 \\
\hline 2018 & 66.86 \\
\hline 2019 & 77.55 \\
\hline
\end{tabular}

construction should consider. The externality is corrected, and the resource allocation can return to the effective state. The quantitative analysis of noise cost of high-speed railway in this paper is helpful to the management to make correct decisions and realize the best combination of economic and environmental benefits of enterprises.

\section{References}

1. European commission. Handbook on the external costs of transport (Version2019) [M] (2019)

2. Di Jing. Research on the theory and method of external cost calculation of China's transportation system (D). Beijing: Beijing Jiao tong University, (2010)

3. Koelega, H.S. Envionmental Annoyance: characterization, measurement and control[M]. Amsterdam: Elsevier (1987)

4. Babisch, W., The Noise/Stress Concept, Risk Assessments and Research Needs, Noise Health, 2002,4(16), 1-11

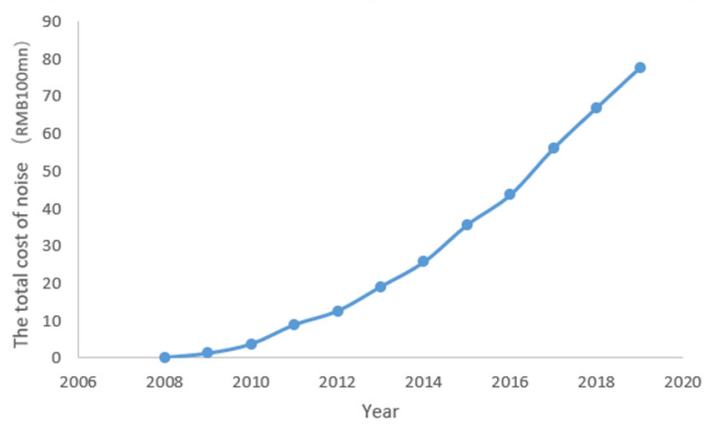

5. Vienneau, D., Perez, L., Schindler, C. The relationship between traffic noise exposure and ischemic heart disease: a meta-analysis. 42nd International Congress and Exposition on Noise Control Engineering. Proceedings of INTER-NOISE, Innsbruck, pp. 5070-5076 (2013)

6. World Bank Open Data. https://data.worldbank.org.cn/

7. China Statistical Yearbook. State Statistical Bureau. http://www.stats.gov.cn/tjsj/ndsj/

Fig. 2. The total noise cost of high-speed railway.

From Figure 2, the total noise cost of high-speed railway has been increasing year by year from 2008 to 2019. Unlike the average noise cost, the increase rate of total noise cost is very obvious. Based on the analysis of the above data, it can be found that in the initial stage of high-speed railway operation in China, the passenger turnover is small, so the total noise cost is low. With the rapid development of high-speed railway, the operating mileage of high-speed railway increases year by year, the passenger turnover increases significantly, so the total noise cost of high-speed railway also increases significantly.

\section{Summary}

This paper mainly analyses the external cost of noise in China's high-speed railway. Based on the unit value transfer method, the calculation model of noise external cost of high-speed railway in China is established. The huge external cost indicates that the negative impact of high-speed railway noise cannot be ignored. All stakeholders of high-speed railway project should fully realize the harm and huge economic loss caused by the existence of external cost to the society. How to reduce the noise influence and internalize the negative external cost of high-speed railway noise should be the key problem that the decision makers of high-speed railway 